\title{
Confirmación de Streptococcus suis en Chile
}

\author{
Pedro Alarcón L., Pamela Araya R., Carolina Aguayo, Jorge Fernández, \\ Vijna Illesca, Angela Zaror y Alejandra Vaquero
}

\section{Laboratory Confirmation of Streptococcus suis in Chile}

(Artículo relacionado en página 557)

\section{Antecedentes}

$S$ treptococcus suis pertenece a la familia Streptococcaceae. Es una cocácea grampositiva, que puede presentar $\alpha$ o $\beta$ hemólisis en agar sangre de cordero; corresponde a los grupos R, S o T de Lancefield, aunque algunos pueden reaccionar con el grupo D. Streptococcus suis posee cápsula por lo cual se identifican 35 serotipos, siendo el serotipo 2 por sus factores de virulencia el que se aísla con mayor frecuencia en cuadros de meningitis, tanto en cerdos como en humanos ${ }^{1,2}$. Este microorganismo se encuentra en el tracto respiratorio, amígdalas, cavidad nasal del cerdo y en su tracto genital y digestivo; afecta a los cerdos produciendo brotes epidémicos de meningoencefalitis, endocarditis, poliserositis y neumonitis ${ }^{3,4}$. Es una enfermedad veterinaria endémica en países con una gran industria de cerdos y corresponde a una zoonosis emergente, siendo un agente que se ha detectado en franco aumento en países europeos ${ }^{5,6}$, Hong Kong ${ }^{7}$ y en el Sudeste asiático ${ }^{8}$. Según la literatura científica, Streptococcus suis puede ser la causa más común en el Sudeste asiático de meningitis estreptocóccica en el adulto?.

La mayoría de los casos de infección en humanos corresponden a manipuladores de cerdos, personal que los alimenta, particularmente los que trabajan en mataderos, que se contaminan por carne cruda o vísceras de los animales, manipulan o comen carne no bien cocida de cerdo ${ }^{10,11}$. La transmisión se produce a través de lesiones en la piel, en mucosa bucal y cavidad nasal o por ingestión de productos contaminados ${ }^{12-14}$.

El cuadro clínico en el humano se manifiesta como una meningitis purulenta, shock séptico, con falla multiorgánica, endocarditis, neumonía, artritis y peritonitis ${ }^{15}$. Entre los síntomas que aparecen en la forma aguda de la meningitis se encuentran la fiebre alta, cefalea, escalofríos, náuseas, vómitos y vértigo, seguidos por uno o más de los siguientes: pérdida de la audición, ataxia cerebelosa, petequias, dolor articular y parálisis facial, rigidez de nuca, mialgia intensa, equimosis y rabdomiolisis ${ }^{16-20}$. El síntoma más destacable es la presencia de desorientación y sordera ${ }^{21}$, siendo éstos los síntomas precoces del cuadro de meningitis ${ }^{8,12}$. Streptococcus suis se puede aislar en hemocultivos. Por lo general responde bien al tratamiento con dosis altas intravenosas de penicilina. Los casos fatales son infrecuentes, pero existen ${ }^{22}$.

\section{Antecedentes de laboratorio}

El Laboratorio de Referencia de cocáceas grampositivas, de la Sección de Bacteriología del Departamento Biomédico Nacional y de Referencia del Instituto de Salud Publica de Chile (ISP) a través de pruebas bioquímicas convencionales, con el apoyo de equipos automatizados mediante espectrometría de masas (MALDI-TOF) y finalmente mediante el secuenciamiento de ARN 16S, ha confirmado la identificación de $S$. suis en cuatro cepas procedentes de casos clínicos.

El laboratorio de Genética Molecular del ISP realizó la tipificación genética de las cepas mediante electroforesis en campo pulsado (EFCP) utilizando la enzima de restricción Apal según protocolo estandarizado. Las imágenes fueron analizadas utilizando el software Bioinformático Bionumerics

\section{Primera detección en Chile}

Fueron dos cepas recibidas desde el Hospital Clínico Regional de Valdivia, en los meses de marzo y mayo de 2012, identificadas presuntivamente como Streptococcus suis. Las muestras recibidas correspondían a dos casos clínicos con diagnóstico de meningitis, uno con 54 años, de sexo femenino, y el segundo con 48 años, de sexo masculino. El análisis molecular demostró la presencia de dos subtipos genéticos distintos CL-APA-SSU-001 y CL-APA-SSU-002, concluyéndose que ambas cepas de S. suis presentaban una similitud de $86,67 \%$ con cinco bandas de diferencia.

\section{Tercera confirmación}

En el mes de julio de 2012, se recibió desde el Hospital Hernán Henríquez Aravena de Temuco, una cepa de LCR, en la que el laboratorio del hospital regional identificó Streptococcus spp. Correspondía a un caso clínico con diagnóstico de meningitis, con 55 años de edad, de sexo
Preparado, con fecha 4 de enero de 2013 por Instituto de Salud Pública de Chile.

Instituto de Salud Pública. Departamento Laboratorio Biomédico Nacional y de Referencia (PAL, PAR, CA, JF). Departamento de Asuntos Científicos (AV). Laboratorio Clínico. Hospital Clínico Regional de Valdivia. Chile (AZ).

Laboratorio Clínico. Hospital Hernán Henríquez Aravena de Temuco. Chile (VI).

Recibido: 2 de septiembre de 2013 


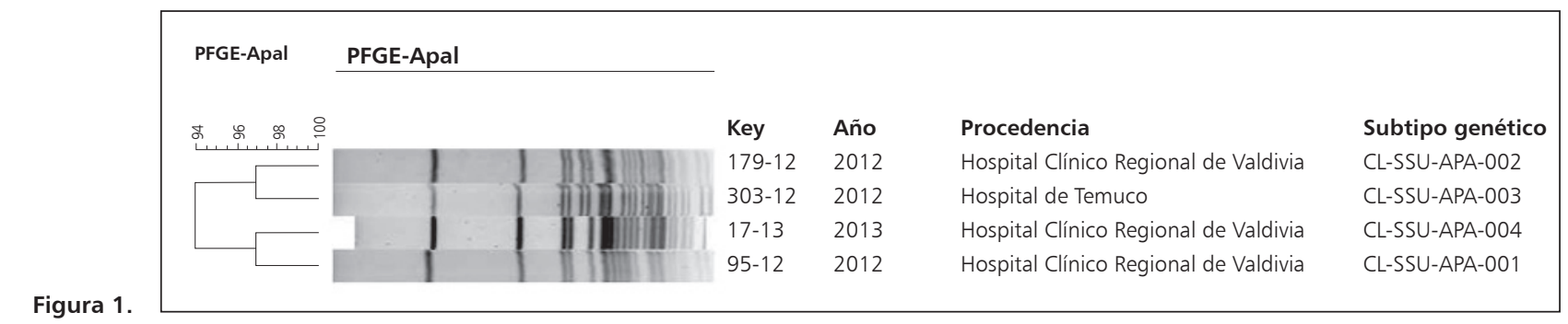

masculino. El Laboratorio de Referencia confirmó la identificación de S. suis y el análisis molecular demostró la presencia del subtipo genético denominado CL-APASSU-003 el cual presenta una similitud de $94,1 \%$ con el subtipo genético identificado anteriormente denominado CL-APA-SSU-002, aislado en el Hospital Clínico Regional de Valdivia, indicando que genéticamente las cepas estarían relacionadas.

\section{Cuarta confirmación}

El 14 de enero de 2013, el Laboratorio de Referencia del ISP, confirmó la cuarta cepa de S. suis. Dicha confirmación correspondió a una cepa derivada por el Hospital Clínico Regional de Valdivia, correspondiente a un caso con diagnóstico de sepsis en un paciente con 47 años de edad, de sexo masculino. El análisis molecular demostró un subtipo genético nuevo denominado CL-APASSU-004 que presentaba $94 \%$ de similitud con el subtipo CL-APA-SSU-001, identificado previamente en una cepa procedente del mismo centro asistencial (Figura 1).

\section{Conclusiones}

Las confirmaciones de aislados de $S$. suis realizadas a la fecha corresponden a cepas de la misma zona geográfica del país, las que además se encuentran genéticamente relacionadas.

Por lo anterior, se estima necesario reforzar el diagnóstico de laboratorio, tanto en esta zona como en el resto del país. Asimismo complementar con estudios de las potenciales fuentes de la infección, a fin de la establecer relaciones causales.

\section{Referencias bibliográficas}

1.- Versalovic J, Carroll K, Funke G. Streptococcus. Manual of Clinical Microbiology, 10th edition. 2011; 20: 331-49.

2.- Winn W, Allen S, Janda W, Koneman E, Procop G, Woods G. Grampositive cocci. Koneman's Color Atlas and Textbook of Diagnostic Microbiology, 6th edition. 2007; 13: 674-764.

3.- Windsor R S, Elliott S D. Streptococcal infection in young pigs. IV. An outbreak of streptococcal meningitis in weaned pigs. J Hyg (Lond) 1975: 69-78.

4.- Anonymous. Porcine streptococci causing meningitis and septicaemia in man. Lancet 1975; 1: 1286-8.

5.- Lutticken R, Temme N, Hahn G, Bartelheimer E W. Meningitis caused by Streptococcus suis: case report and review of the literature. Infection 1986; 14: 181-5.

6.- Colaert J, Allewaert M, Magerman H, Vandeven J, Vandepitte J. Streptococcus suis meningitis in man. First reported observation in Belgium. Acta Clin Belg 1985; 40: 314-7.

7.- $\quad$ Chau P Y, Huang C Y, Kay R. Streptococcus suis meningitis. An important underdiagnosed disease in Hong Kong. Med J Aust 1983; 1: 414-6, 417.

8.- World Health Organization Regional Office for the Western Pacific. Outbreak associated with Streptococcus suis in pigs in China: update 2005. http://www.who.int/zoonoses/outbreaks/ zoonosesoutbreaksuis 1/en/index.html

9.- Suankratay C, Intalapoporn P, Nunthapisud P, Arunyingmongkol K, Wilde H. Streptococcus suis meningitis in Thailand. Southeast Asian J Trop Med Public Health 2004; 35: 868-76.

10.- Clifton-Hadley F A, Alexander T J, Enright M R, Guise J. Monitoring herds for Streptococcus suis type 2 sampling tonsils of slaughters pigs. Vet Rec 1984; 115: 562-4.

11.- Breton J, Mitchell W R, Rosendal S. Streptococcus suis in slaughter pigs and abattoir workers. Can J Vet Res 1986; 50: 338-41.

12.- Willenburg K, Sentochnik D E, Zadoks R N. Human Streptococcus suis in the United States. New Engl J Med 2006; 354:1325.

13.- Francois B, Gissot V, Ploy M C, Vignon P. Recurrent septic shock due to Streptococcus suis. J Clin Microbiol 1998; 36: 2395.

14.- Yuan H, Jing H, Chen Z, Zheng H, Zhu X, Wang H, et al. Human Streptococcus suis outbreak, Sichuan, China. Emerg Infect Dis 2006; 12: 914-20.

15.- Du Y P, Qian W J, Xu G B. Investigation on 8 human cases with meningitis caused by Streptococcus suis type 2. Chin J Prev Med 2000; 34: 305.
16.- Fongcom A, Pruksakorn S, Mongkol R, Tharavichitkul P, Yoonim N. Streptococcus suis infection in northern Thailand. J Med Assoc Thai 2001; 84: 1502-8.

17.- Matsuo H, Sakamoto S. Purulent meningitis caused by Streptococcus suis in a pig breeder. Kansenshogaku Zasshi 2003; 77: 340-2.

18.- Rosenkranz M, Elsner H A, Sturenburg H J, Weiller C, Rother J, Sobottka I. Streptococcus suis meningitis and septicemia contracted from a wild boar in Germany. J Neurol 2003; 250: 869-70.

19.- Tambyah PA, Kumarasinghe G, Chan H L, Lee K O. Streptococcus suis infection complicated by purpura fulminans and rhabdomyolysis: case report and review. Clin Infect Dis 1999; 24: 710-2.

20.- Zhang X Y, Ding J Q, Qin H P. Clinical analysis of 22 cases of the disease contracted both by man and pigs with swine streptococcus infection. J Trop Med 2002; 2: 361-3.

21.- Shneerson J M, Chattopadhyay B, Murphy M F, Faecet I W. Permanent perceptive deafness due to Streptococcus suis type II infection. J Laryngol Otol 1980; 94: 425-7.

22.- Sriskandan S, Slater J D. Invasive disease and toxic shock due to zoonotic Streptococcus suis; an emerging infection in the East? PLoS Med 2006; 3: e187. 